En agissant ainsi, on associera heureusement les quatre grands facteurs d'assainissement du lait: l'éducation, le stimulant économique, la prévention, la répression.

5 . Au moment où l'industrie laitière traverse une crise particulièrement grave, il est temps de rappeler, avec insistance, que le meilleur remède à la sous-consommation dont se plaignent producteurs et industriels laitiers, la meilleure propagande consistent, sans nul doute, à faire l'emporter la qualité sur la quantité, cette qualité devant être garantie par un contrôle officiel.

6. Imposer le contrôle hygiénique du lait n'est nullement attenter à la liberté du commerce, c'est simplement placer l'intérêt général au-dessus de certains intérêts particuliers, d'ailleurs mal compris. Une loi fort importante (loi du 7 juillet 1933) vient de généraliser, en France, l'inspection hygiénique des viandes et produits dérivés. Il est nécessaire qu'un contrôle semblable soit institué pour le lait.

\title{
LA TENSION SUPERFICIELLE DU LAIT
}

\section{par}

\section{Dr W. KOPACZEWSKI}

Le travail de G. BeLle, publié dans ce périodique, sur le même sujet [1] me donne l'occasion de rappeler les recherches personnelles sur les caractères physico-chimiques du lait et de les compléter.

En effet, jusqu'à 1925, date à laquelle nous avons publié les résultats globaux de nos recherches, les constantes physiques du lait n'ont fait l'objet que de rares déterminations et à l'aide de méthodes bien approximatives [2]. En ce qui concerne la tension superficielle, par exemple, on ne pouvait citer que quelques mesures de Imbert et Duclos (1905), de Kobler (1908), de Meillè̀re, et de WWEDENSKY (1925), effectuées à l'aide d'un compte-gouttes, ne tenant compte ni de la température, ni de la densité.

En 1925, nous avons utilisé, pour cette mesure, le tonomètre de notre construetion et en nous basant sur une trentaine de déterminations, effectuées sur les échantillons du lait du commerce parisien, ainsi que sur le lait aussitôt après la traite, nous avons donné les earactères physico-chimiques suivants du lait de vache :

\begin{tabular}{|c|c|}
\hline c. & 1,030 \\
\hline Viscosité à $15^{\circ} \mathrm{C}$. & 1,75 \\
\hline Tension superficielle & 52,8 dynes. $\mathrm{cm}^{-1}$ \\
\hline Conductibilité élèctrique & $86,0.10 .4$ \\
\hline
\end{tabular}

Nous avons ajouté, de plus, que ces caractères sont relativement fixes, et permettent de contrôler la pureté du lait de vache.

Ces recherches ont échappé à G. BeLle. 
Nous allons les compléter par quelques déterminations anciennes restées dans notre documentation personnelle.

10 Variation de La tension SUPERFicielle dU LAIT, SElON LA Provenance, - Nous réunissons dans le tableau ci-dessous les principaux résultats (tableau I).

TABLEAU I.

Tension superficielle selon la provenance du lait.

Provenance

A la ferme (A)

Maggi

Maggi

Maggi

Maggi

Hauser

Hauser

Crémier (A)

Crémier (B)

Crémier (C)

Crémier (D)
Température

Tension superficielle en dynes $\mathrm{Cm}-\mathrm{I}$

$15^{\circ} \mathrm{C}$.
$15^{\circ} \mathrm{C}$.
$15^{\circ} \mathrm{C}$.
$15^{\circ} \mathrm{C}$.
$15^{\circ} \mathrm{C}$.
$15^{\circ} \mathrm{C}$.
$15^{\circ} \mathrm{C}$.
$15^{\circ} \mathrm{C}$.
$15^{\circ} \mathrm{C}$.
$15^{\circ} \mathrm{C}$.
$15^{\circ} \mathrm{C}$.
$15^{\circ} \mathrm{C}$.

53,7

54,2

54,9

53,3

53,6

53,4

54,1

53,4

54,4

54,2

55,0

51,7
Densité

1,027

1,028

1,030

1,029

1,030

1,029

1,030

1,029

1,030

1,027

1,030

1,027

Ainsi, les valeurs extrêmes sont les suivantes : 51,7 et 55,0 dynes $\mathrm{cm}^{-1}$; la moyenne de toutes nos déterminations (37 échantillons) est de 52,8 dynes $\mathrm{cm}^{-1}$. Si l'on tient compte de l'écart de la température entre 15 et $18^{\circ} \mathrm{C}$., nos chiffres peuvent être considérés comme très analogues à ceux publiés par BeLLE sur le lait marocain. Soulignons, de plus, que ce léger écart peut être expliqué par les incertitudes de la méthode d'arrachement qui a été adoptée par ce dernier auteur, quoiqu'il semble que toutes les précautions ont été prises par lui pour les éviter (temps de détermination, propreté des vases, etc.) ; néanmoins, cette méthode doit être surtout employée pour étudier l'évolution d'un processus physique et non les valeurs de la tension superficielle des liquides mal équilibrés, tels que les complexes colloïdaux. Nous avons longuement insisté sur ces points dans notre "Traité de Biocolloïdologie" (voir, t. I, p. 425) et dans un mémoire spécial consacré à la tension superficielle [3].

Par contre, nos mesures semblent indiquer que la tension superficielle des divers échantillons du lait de vache, à condition qu'il ne soit point falsifié, est fixe.

$2^{\circ}$ TeNSION SUPERFICIELLE DU LAIT DE VACHE EN FONCTION DE TEMPS. - Nous avons constaté des variations suivantes (tableau II). 
TABLEAU II.

Tension superficielle en fonction de temps a $15^{\circ} \mathrm{C}$.

Echantillons

Tension superficielle après $x$ heures :

\begin{tabular}{|c|c|c|c|c|c|c|}
\hline & 0 & 6 & 12 & 18 & 24 & 36 \\
\hline & 53,6 & 54,1 & 54,7 & 55,0 & 55,5 & 55,9 \\
\hline II & 55,1 & 55,4 & 55,9 & 55,9 & 56,6 & 56,9 \\
\hline
\end{tabular}

Nous constatons done une graduelle augmentation de la tension superficielle, au fur et à mesure de son vieillissement. Remarquons que cette conservation a été effectuée dans des tubes paraffinés placés dans un thermostat réglé à $15^{\circ} \mathrm{C}$. Point intéressant, sur lequel nous nous proposons de revenir, le lait coagulé, gélifié comme il serait plus correct de le dire, possède, après la centrifugation, une tension superficielle notablement plus basse : 49,7 dynes $\mathrm{cm}^{-1}$. Ces résultats ne s'accordent pas avec ceux que nous trouvons dans la publication de BELLE; d'après eet auteur on assisterait à une baisse progressive de la tension superficielle durant 24 heures. Peut-être ses expériences ont trait à des laits plus frais dont la gélification est, évidemment, plus tardive ; peut-être aussi, la méthode d'arrachement en est responsable.

3o Tension superficielle du lait et la dilution. - Nous avons essayé de fixer le degré d'hydratation du lait par la mesure de la tension superficielle (tableau III).

TABLEAU III.

Tensiōn superficielle du lait et sa dilution, à $15^{\circ} \mathrm{C}$.

Degré de dilution

Pur

Dilué au $1 / 20^{e} \ldots \ldots \ldots \ldots \ldots \ldots$

Dilué au $1 / 10^{\mathrm{e}} \ldots \ldots \ldots \ldots \ldots$.

Dilué au $1 / 5^{\mathrm{e}} \ldots \ldots \ldots \ldots \ldots \ldots$
Tension superficielle

53,7

54,2

53,9

53,7
Densité

1,031

1,029

1,026

1,014

On voit, par conséquent, que la tension superficielle du lait ne peut servir à la détermination de son degré d'hydratation : le lait, comme tous les liquides organiques, possède un pouvoir régulateur énergique qui lui permet de s'opposer à des actions extérieures, tendant à modifier ses caractéristiques physiques. Nous renvoyons les lecteurs qui s'intéressent à ce sujet, à nos recherches sur le pouvoir-régulateur des colloïdes, en général [4].

$4^{\circ}$ Tension SUPERficielle du latt de Vache ÉCRémé. - Voici quelques chiffres (tableau IV).

\section{TABLEAU IV.}

Tension superficielle du lait écrémé à $15^{0} \mathrm{C}$.

Provenance
Tension superficielle du lait pur écrémé partiellement par centrifugation

\begin{tabular}{|c|c|c|}
\hline Crémerie (A) $\ldots \ldots \ldots \ldots \ldots$ & 54,48 & 54,94 \\
\hline Maggi $\ldots \ldots \ldots \ldots \ldots \ldots$ & 54,9 & 55,1 \\
\hline Ferme............... & 54,2 & 55,8 \\
\hline
\end{tabular}


Après la centrifugation, la tension superficielle du lait augmente légèrement. Ceci semble indiquer que la présence des matières grasses en suspension n'influe pas sur ce caractère phy' sique du lait.

5o Tension sUperficielle et agitation. - L'agitation augmente la tension superficielle du lait. Voici quelques chiffres (tableau V).

TABLEAU V.

Agitation et tension superficielle du lait à $15^{\circ} \mathrm{C}$.

Echantillons

Tension superficielle du lait agité durant pur après un repos de 12 heures 5 minutes

I $\ldots \ldots \ldots \ldots \ldots \ldots$

52,5

54,4

II $\ldots \ldots \ldots \ldots \ldots \ldots$

55,4

56,8

III

54,1

56,7

Il convient, toutefois, de remarquer que ces mesures, effectuées sur un liquide non équilibré, n'ont pas une signification bien précise.

\section{Conclusions}

1. La tension superficielle du lait pur est fixe ; elle s'établit vers 53 dynes/cm.

2. La dilution n'affecte pas beaucoup cette constante capillaire du lait ; le pouvoir régulateur joue dans ce cas, comme il joue dans les cas des colloïdes, en général.

3. La tension superficielle du lait écrémé n'est pas sensiblement différente du lait complet.

$4^{\circ} \mathrm{Au}$ cours de vieillissement le lait présente une augmentation nette de sa tension superficielle.

5. L'agitation du lait s'accompagne d'une augmentation de sa tension superficielle.

\section{BIBLIOGRAPHIE.}

[1] G. Belle. Le Lait, t. XVI, 1936, p. 13.

[2] W. Kopaczewski. Etat colloïdal et Industrie, Paris, 1925. Librairie polytechnique Béranger, éditeurs, t. I, p. 133-134.

[3] W. Kopaczewski. Traité de Biocolloïdologie, Paris, 1930-1935, t. I, p. 449.

W. Kopaczewski. Protoplasma, t. XIX, 1933, p. 255. Rôle de la tension superficielle en biologie (Bibliographie).

[4] W. Kopaczewski. Protoplasma, t. IX, 1930, p. 598. Le pouvoir-tampon du sérum. Traité de Biocolloïdologie, t. III, p. 428. Gauthier-Villars, éditeurs. 